\title{
Regionale Sterblichkeitsunterschiede in der Schweiz: ein nicht ganz einfach zu bestimmender Indikator für regional ungleiche Lebenschancen
}

\section{Zusammenfassung}

Die Lebenserwartung bzw. das relative Sterberisiko ist ein wichtiger Indikator für die Lebenschancen in einer Region. Für aussagekräftige Vergleiche von Mortalitätsmaßen müssen die Unterschiede in der regionalen Altersstruktur, aber auch zwischen Mann und Frau berücksichtigt werden. Daneben müssen in der Schweiz mehrere Zähler-Nenner-Probleme gelöst werden, die durch unterschiedliche Wohnsitzdefinitionen und den Einbezug der Ausländer bedingt sind. Für die 35- bis 64jährigen Schweizer Bürger ergeben sich erhebliche regionale Disparitäten, die einem Unterschied von mehreren Jahren Lebenserwartung entsprechen. Bei den Männern sind die geographischen Unterschiede ausgeprägter und Ausdruck eines gesundheitsgefährdenderen Verhaltens. Erhöhte Mortalität betrifft vor allem die Suisse romande und die Großstädte. Regionale Sterblichkeitsunterschiede sind Ausdruck kultureller und sozialer Lebensbedingungen. Eine sorgfältige Analyse der Unterschiede und Trends muß daher die Regionalforschung beschäftigen.

\section{Summary}

Life expectancy is a major public health issue and an important measure of regional living conditions. Valid analyses of mortality should not only consider regional age structure and differences between males and females but also several incongruities between numerator and denominator, arising from discordant definitions of domicile and from less reliable mortality figures for the foreigners. For Swiss citizens aged 35 to 64 years the patterns of all-cause mortality in Switzerland present remarkable regional disparities, amounting to several years of life expectancy. Geographical variation is more important in males than in females, corresponding to the riskier health behaviour of men. Unfavourable mortality figures were revealed for French-speaking regions and the big cities. Regional mortality figures and trends reflect cultural and social circumstances. Carefully analysed they offer a lot of information to the regional scientist.

\section{Einleitung}

Die mittlere Lebenserwartung bzw. das relative Sterberisiko ist ein zentrales $\mathrm{Ma} \beta$ für die Lebenschancen und Lebensbedingungen in einem Land oder einer Region. Eine optimale Gestaltung dieser Lebensbedingungen sollte daher nicht nur für Prävention und Gesundheitspolitik, sondern auch für die Regionalpolitik zu den vorrangigen Zielen gehören.

Seltsamerweise hat dieser Themenbereich in der Geographie bis heute nur wenig Beachtung gefunden, sogar im angelsächsischen Raum (CLIFF \& HAGGETT 1988).
Medizinische Geographie oder geographische Epidemiologie (E. = Lehre von der Verteilung der Gesundheitszustände und ihrer Determinanten, GUTZWILLER \& JEANNERET 1996) sind in der Schweiz - abgesehen von einer Blüte zu Beginn des Jahrhunderts, als sogar Auswertungen auf Bezirksebene durchgeführt wurden' historisch kaum verwurzelt und bezeichnenderweise auch kein Thema für Geographiebücher. Auch in den meisten anderen europäischen Ländern sind regionale Sterblichkeitsunterschiede nur sehr selten von Geographen analysiert worden (eine löbliche Ausnahme stellt LÜTZELER 1994 dar).

In der Medizin erweckten geographische Unterschiede in der Häufigkeit chronischer Krankheiten seit den 60er Jahren zunehmend Interesse, was vor dem Hintergrund des aufblühenden Risikofaktorenmodells in den meisten Ländern zur Publikation von Krebsatlanten geführt hat (HOWE 1986, BOYLE et al. 1989), auch in der Schweiz, allerdings nur auf Kantonsebene und kartographisch mangelhaft (BROOKE 1975, BUNDESAMT FÜR STATISTIK 1984). Die Geographie der Gesamtmortalität wurde hingegen nicht zum Forschungsgegenstand, wohl, weil sie vom epidemiologischen Risikofaktorenmodell her wenig versprach und Fragen der allgemeinen Lebensqualität für die Medizin damals kaum ein Thema waren (der breitere Public-Health-Ansatz hat sich bei uns erst in den letzten Jahren verbreitet).

In der Schweiz wurden erst in den 80er Jahren Karten zur Geographie der Gesamtmortalität publiziert, aber nur auf Kantonsebene (BISIG \& PACCAUD 1987) oder nur für die Männer (BUNDESAMT FÜR STATISTIK 1981) bzw. für beide Geschlechter zusammen (SCHULER et al. 1984). Seit kurzem liegt nun erstmals ein Werk vor, das die regionalen Unterschiede im Zeitraum 1970-1990 für die Gesamtmortalität und die wichtigsten Todesursachen ausführlich dokumentiert (SCHÜLER \& BOPP 1997).

Die Sterberaten in der Schweiz gehören im europäischen Umfeld zu den niedrigsten, oder mit anderen Worten ausgedrückt, die Lebenserwartung ist eine der höchsten. Die Frauen in der Schweiz teilen ihre günstige Position nur mit Frankreich, die Männer mit Spanien, Griechenland und Schweden, bleiben aber hinter Island zurück. Aller-

Matthias Bopp, Dr., Institut für Sozial- und Präventivmedizin der Universität Zürich, Sumatrastraße 30, 8006 Zürich 
dings täuschen Ländervergleiche: Baden-Württemberg und Vorarlberg/Tirol haben innerhalb Deutschlands bzw. Österreichs eine besonders niedrige Mortalität, kaum höher als ihre Schweizer Nachbarschaft (sANS et al. 1997). Zudem bestehen innerhalb der Schweiz erhebliche regionale Unterschiede (SCHÜLER \& BOPP 1997), die sich meist über längere Zeit zurückverfolgen lassen. Dies ist bemerkenswert, weil die Sterberaten im 20. Jahrhundert massiv zurückgegangen sind - in der Altersgruppe 35-64 in nur 25 Jahren um über einen Drittel (sowohl bei den Männern als bei den Frauen $-37 \%$ zwischen 1969/70 und 1993/94).

\section{Methoden}

Die Berechnung von validen Sterblichkeitsmaßen unterliegt einigen Besonderheiten, die dem Geographen nicht unbedingt vertraut sind, weshalb sie hier kurz skizziert werden.

\section{a) Korrektur für Abweichungen im Altersaufbau}

Die Wahrscheinlichkeit, innerhalb des nächsten Kalenderjahres zu sterben, nimmt mit steigendem Alter fast exponentiell zu. Deshalb wäre es grob irreführend, einfach die Todesfallzahlen durch die Regionsbevölkerung zu dividieren. Vielmehr muß der je nach Region unterschiedliche Altersaufbau der Bevölkerung bei der Berechnung berücksichtigt werden. Dazu bestimmt man für eine definierte Zeitperiode und Region zuerst altersspezifische Sterberaten (Sterbefälle der Altersgruppe i pro 100000 Einwohner der Altersgruppe i), die dann mit einer vorgegebenen Altersstruktur gewichtet und aufsummiert werden. Dieser Vorgang wird als Altersstandardisierung bezeichnet. Als guter Kompromiß bezüglich Aufwand und Genauigkeit hat sich dabei die Verwendung von 5-Jahres-Altersklassen eingebürgert, wobei die über 85 jährigen nicht mehr weiter aufgegliedert werden (BRESLOW \& DAY 1987).

\section{b) Ausrichtung auf das Landesmittel}

Aus didaktischen Gründen wird für vergleichende Mortalitätsberechnungen anstelle von altersstandardisierten Sterberaten meist der (alters)standardisierte Mortalitätsquotient (Standardized Mortality Ratio, SMR) vorgezogen. Die SMR berechnet sich, indem die Zahl der in einer bestimmten Region aufgetretenen Sterbefälle dividiert wird durch die Summe der Todesfälle, die unter Zugrundelegung der altersspezifischen Sterberaten der Gesamtschweiz in jeder Altersgruppe zu erwarten wären; für das Landesmittel resultiert stets der Wert 1 oder - als Prozent ausgedrückt - 100. Die SMR drückt ein relatives Risiko aus: Eine SMR von 150 bedeutet ein gegenüber dem Landesmittel um 50\% erhöhtes Sterberisiko.

\section{c) Berechnung der statistischen Signifikanz}

Die mitunter kleinen Fallzahlen führen dazu, daß bevölkerungsschwache Regionen zu Extremwerten («Zufallsschwankungen») neigen. Um das Gewicht von Ausreißern zu relativieren, wird die Wahrscheinlichkeit berechnet, daß das vorliegende Resultat allein durch Zufall eintritt. Beträgt diese Wahrscheinlichkeit weniger als $5 \%$, gilt das Resultat als statistisch signifikant. (Dies bedeutet nicht unbedingt, daß der Befund auch inhaltlich bedeutsam oder gesichert ist.)

\section{d) Konzept der Langfristigkeit}

Viele Krankheiten unterliegen einer oft jahrzehntelangen Latenzzeit (Zeit zwischen Einwirkung einer Schädigung und Auftreten erkennbarer Symptome); weitere Zeit verstreicht zwischen Diagnose und allfällig durch diese Krankheit verursachtem Tod. Was sich heute als geographisches Muster in der Todesfallstatistik äußert, ist daher meist das Abbild vergangener Lebensbedingungen und Verhaltensweisen. Da sich diese jedoch nur sehr langsam ändern, haben die geographischen Muster chronischer Krankheiten meist ein beträchtliches Persistenzvermögen. Dieser Umstand ist bei der Beurteilung von Risikoabweichungen in bevölkerungsschwachen Gebieten von zentraler Bedeutung (BOPP \& SCHÜLER 1997).

\section{e) Unterschiede zwischen Mann und Frau}

Frauen haben in allen entwickelten Ländern eine deutlich höhere Lebenserwartung als Männer. Gemäß der aus den Sterbedaten für 1988-93 abgeleiteten Sterbetafel für die Schweiz (WANNER 1996) erreichen von 100000 neugeborenen Knaben fast 20000 ihren 65. Geburtstag nicht, von 100000 Mädchen sind es dagegen nur 10000 . Wegen der höheren Sterblichkeit der Männer würden diese bei einer Zusammenfassung beider Geschlechter die Sterblichkeitsmuster dominieren. Deshalb, und weil Übereinstimmung der geographischen Mortalitätsmuster von Mann und Frau eher die Ausnahme als die Regel ist (SCHÜLER \& BOPP 1997), sollte man die Geschlechter stets getrennt analysieren.

\section{f) Zähler und Nenner aus verschiedenen Datenbasen}

Als Datenquelle mit hoher geographischer Auflösung steht in der Schweiz für die Altersstruktur der regionalen Bevölkerungen einzig die Volkszählung zur Verfügung. Während die Wohnortdefinition der Todesursachenstatistik seit 1981 auf dem zivilrechtlichen Begriff fußt (Wohnort $=$ Gemeinde, in der die Schriften deponiert sind), verwendet die Volkszählung traditionell den wirtschaftlichen Wohnsitzbegriff. Bei der Bestimmung der Gesamtsterblichkeit ergeben sich in erster Linie Probleme wegen der Heiminsassen (in der Volkszählung zur Standortgemeinde des Heims gezählt, in der Todesursachenstatistik zur Herkunftsgemeinde), aber auch wegen 
der Saisonniers (nur in der Volkszählung erfaßt, wegen des Dezember-Zeitpunkts der Volkszählung v. a. für Winterkurorte von Bedeutung). Für einige Regionen ergeben sich daraus erhebliche Abweichungen (SCHÜLER \& BOPP 1997). Eine weitere Verzerrung schleicht sich durch die Altersberechnung der Volkszählung ein, die traditionell durch Subtraktion des Geburtsjahrgangs vom Volkszählungsjahr erfolgt, wodurch jedem Individuum rund ein Monat «geschenkt» wird, was in den höchsten Altersklassen nicht belanglos ist.

Die Volkszählung 1990 brachte gegenüber ihren Vorgängerinnen zwei für die Entschärfung der erwähnten Probleme entscheidende Neuerungen: erstmals wurde nicht nur das Geburtsjahr, sondern auch der exakte Geburtstag erhoben, was eine exakte Altersberechnung erlaubt. Wichtiger ist aber der Versuch, Personen mit Abweichungen zwischen zivilrechtlicher und wirtschaftlicher Wohngemeinde getrennt auszuweisen. Im Gegensatz zu den publizierten Tabellen, die weiterhin auf dem wirtschaftlichen Wohnsitzbegriff und aufgerundeten Altersjahren beruhen, kann man aus den Individualdaten für jede Gemeinde die Altersstruktur der Bevölkerung nach dem zivilrechtlichen Wohnsitzbegriff bestimmen und die daraus resultierenden Unterschiede der Mortalitätsmaße illustrieren (Tab. 1). Bei der traditionellen Vorgehensweise errechnet sich für die über 65jährigen Frauen in der Stadt Zürich ein um fast 10\% erhöhtes Sterberisiko, das sich ungünstig von demjenigen in den Städten Basel und Bern oder in der Region Schwarzwasser abhebt, ganz zu schweigen vom Vergleich mit den unterdurchschnittlichen Risiken in den Regionen Weinland und Untersee/Rhein. Dieser Eindruck bleibt auch beim Ausschluß der - in dieser Altersgruppe nicht sehr zahlreichen - Ausländerinnen bestehen. Verwendet man aber auch für den Nenner den zivilrechtlichen Wohnsitzbegriff, so ergibt sich ein völlig anderes Bild: Das Sterberisiko der über 65jährigen Frauen in der Stadt Zürich ist gegenüber dem Landes- mittel nur noch leicht erhöht und unterscheidet sich nicht von demjenigen in Basel oder im Weinland, während Untersee/Rhein und Schwarzwasser weit ungünstiger erscheinen.

\section{Kartographie}

Die hier abgebildeten Karten wurden mit der am Geographischen Institut der Universität Zürich entwickelten Kartographie-Software PS-COPAM (HERZOG 1988) erstellt. Das Prinzip der stufenlosen Darstellung von Datenwerten ist besonders geeignet, sowohl Gradienten als auch echte Trennlinien adäquat zu visualisieren. Außerdem können mit dieser Software Signifikanzangaben in die Karten eingebaut werden. Um das visuelle Übergewicht der dünn besiedelten Alpengebiete zu reduzieren, werden unbewohnte Gebiete nicht eingefärbt (BOPP 1989).

\section{Resultate}

Im Gegensatz und als Ergänzung zur Darstellung im Atlas der Krebsmortalität in der Schweiz 1970-1990 (SCHÜLER \& BOPP 1997) beziehen sich die Karten in diesem Heft auf die Altersgruppe 35-64 Jahre. (Die nicht sehr zahlreichen Tode vor dem 35. Geburtstag werden hier wegen ihres deutlich abweichenden Todesursachenprofils - geringe Bedeutung chronischer Krankheiten, dafür mehr Unfälle, Aids und Suizid - ausgeklammert. Die Tode der über 65 jährigen sind zwar weit zahlreicher als diejenigen vor dem 65 . Geburtstag, aber von der Perspektive der Prävention her viel weniger aussagekräftig.)

Eine je nach Region verschieden gravierende Verzerrung ergibt sich durch den Einbezug der Ausländer: In der

Tab. 1 Altersstandardisierte Mortalitätsquotienten 1989-92 der über 65jährigen Frauen für ausgewählte Teilgebiete und Bevölkerungsdefinitionen (Schweiz = 100).

\begin{tabular}{lccc}
\hline & \multicolumn{2}{l}{$\begin{array}{l}\text { wirtschaftlicher Wohnsitzbegriff } \\
\text { traditionelle Altersberechnung } \\
\text { alle }\end{array}$} & $\begin{array}{c}\text { zivilrechtlicher Wohnsitzbegriff } \\
\text { exakte Altersberechnung } \\
\text { nur Schweizerinnen }\end{array}$ \\
\hline Großstadtgemeinden: & & & 102,1 \\
Zürich & 109,8 & 108,8 & 102,4 \\
Basel & 103,7 & 103,1 & 98,0 \\
Bern & 102,8 & 102,7 & 101,5 \\
MS-Regionen: & & & 124,7 \\
Zürcher Weinland & 93,5 & 93,1 & 110,9 \\
Schwarzwasser & 105,9 & 105,7 & \\
Untersee/Rhein & 95,1 & 94,2 &
\end{tabular}

Datengrundlage: Eigene Berechnungen aus den Individualdaten der Todesursachenstatistik und der Volkszählung 1990 des Bundesamts für Statistik. 
Altersgruppe 35-64 waren um 1990 23\% aller Männer und $14,3 \%$ aller Frauen ausländischer Nationalität. Hinter diesen Zahlen verbergen sich große regionale Unterschiede, die einen Bereich von $4 \%$ bzw. $1,4 \%$ im oberen Emmental bis zu $45,2 \%$ bzw. 32,4\% im Kanton Genf abdecken. Damit ist die Vergleichbarkeit der regionalen Sterblichkeitsmaße nicht mehr garantiert, denn ein Einbezug der Ausländer ergibt generell ein zu günstiges Bild. Da in der Regel nur Gesunde zuwandern und beim Eintreten von schweren Gesundheitsproblemen wohl nicht selten eine Rückkehr ins Herkunftsland stattfindet, fällt das Sterberisiko für Ausländer in der Statistik trotz durchschnittlich niedrigerem Sozialstatus geringer aus als für die Schweizer. In der Epidemiologie ist dieses Phänomen als «healthy worker effect» bekannt (BOUYER et al. 1993). Für die Schweiz als Ganzes fallen die altersspezifischen Mortalitätsraten der Ausländer im Erwerbsalter deutlich geringer aus als für die Schweizer, wohingegen im Rentenalter eine Angleichung zu beobachten ist (Abb. 1). Je nach Gegend weichen die Sterberaten der Ausländer aber stark von diesen Mittelwerten ab. Während etwa in Basel-Stadt das relative Sterberisiko der über 45jährigen Männer kaum vom Landesmittel der Schweizer Bürger abweicht, wird für 50bis 64jährige Ausländer im Aargau eine deutlich niedrigere Mortalität ausgewiesen. Setzt man die Sterberate aller 55- bis 59jährigen Männer in der Schweiz als 100, so ergibt sich für die Ausländer insgesamt ein Wert von 84, in Basel-Stadt einer von 111 (bei den Schweizern: 123), im Aargau ein solcher von 62 (Schweizer: 99). Solche Diskrepanzen sind biologisch wenig plausibel. Als Erklärung viel einleuchtender ist eine je nach Region anderen Prinzipien und Wohnsitzdefinitionen folgende Registrierung der Ausländer in Zähler und Nenner.

Wegen der genannten Probleme wollen wir uns für die regionalen Vergleiche innerhalb der Schweiz auf die Schweizer Bürgerinnen und -Bürger beschränken. Insgesamt ergibt sich für die Periode 1989-92 trotz den erwähnten Vorbehalten eine recht hohe Übereinstimmung mit den im Atlas der Krebsmortalität abgebildeten Mustern für die Gesamtbevölkerung, obwohl nur 17,7\% der Männertode und sogar nur 10,1\% der Frauentode auf 35- bis 64jährige Schweizer Bürger entfallen. Offensichtlich wird das Muster der Gesamtmortalität schon früh vorgezeichnet.

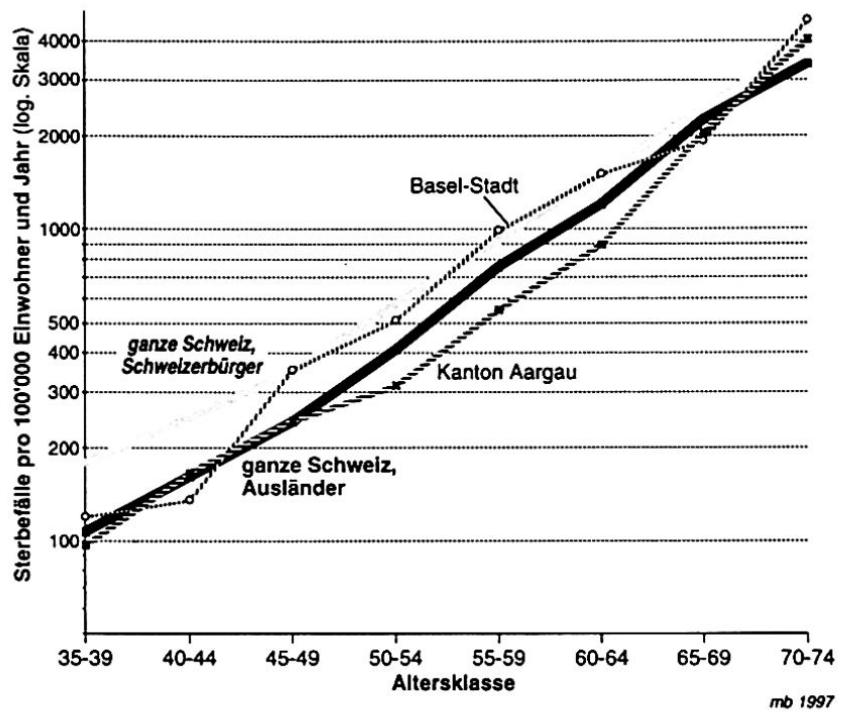

Abb. 1 Altersspezifische Mortalität der 35- bis 74jährigen Männer 1989-92. Schweizer vs. Ausländer total und Ausländer Basel-Stadt vs. Aargau.

Datengrundlage: Eigene Berechnungen aus den Individualdaten der Todesursachenstatistik und der Volkszählung 1990 (zivilrechtliche Wohnsitzdefinition) des Bundesamts für Statistik.

Die folgende Beschreibung der Abweichungen vom Landesmittel berücksichtigt die Muster seit 1970. Die für beide Geschlechter um 1990 niedrige Mortalität für Erlach/Seeland und Aaretal sowie bei den Männern im Luzerner Hinterland und bei den Frauen im Toggenburg bestätigt sich längerfristig so wenig wie bei den Frauen die Erhöhung im Kandertal. Sonst entsprechen die Kartenbilder recht gut den langfristigen Verteilungsmustern.

\section{Männer (Abb. 2)}

Erhöhte Mortalität findet man:

- in einer zusammenhängenden Zone vom Nordjura über den Kanton Neuenburg und Yverdon ins Frei-

Zu Abb. 2 Relatives Sterberisiko der 35- bis 64jährigen Schweizer Männer 1989-92 (siehe rechts).

$\begin{array}{ccc}\text { Statistisch signifikante } & \text { Abweichungen vom Landesmittel sind wie folgt gekennzeichnet: } \\ & + & \text { Risikoerhöhung signifikant auf dem 5\%-Niveau } \\ ++ & \text { Risikoerhöhung signifikant auf dem } 1 \% \text {-Niveau } \\ +++ & \text { Risikoerhöhung signifikant auf dem } 0,5 \% \text {-Niveau } \\ = & \text { niedrigere Mortalität signifikant auf dem } 5 \% \text {-Niveau } \\ == & \text { niedrigere Mortaliät signifikant auf dem } 1 \%-\text { Niveau } \\ === & \text { niedrigere Mortalität signifikant auf dem 0,5\%-Niveau }\end{array}$




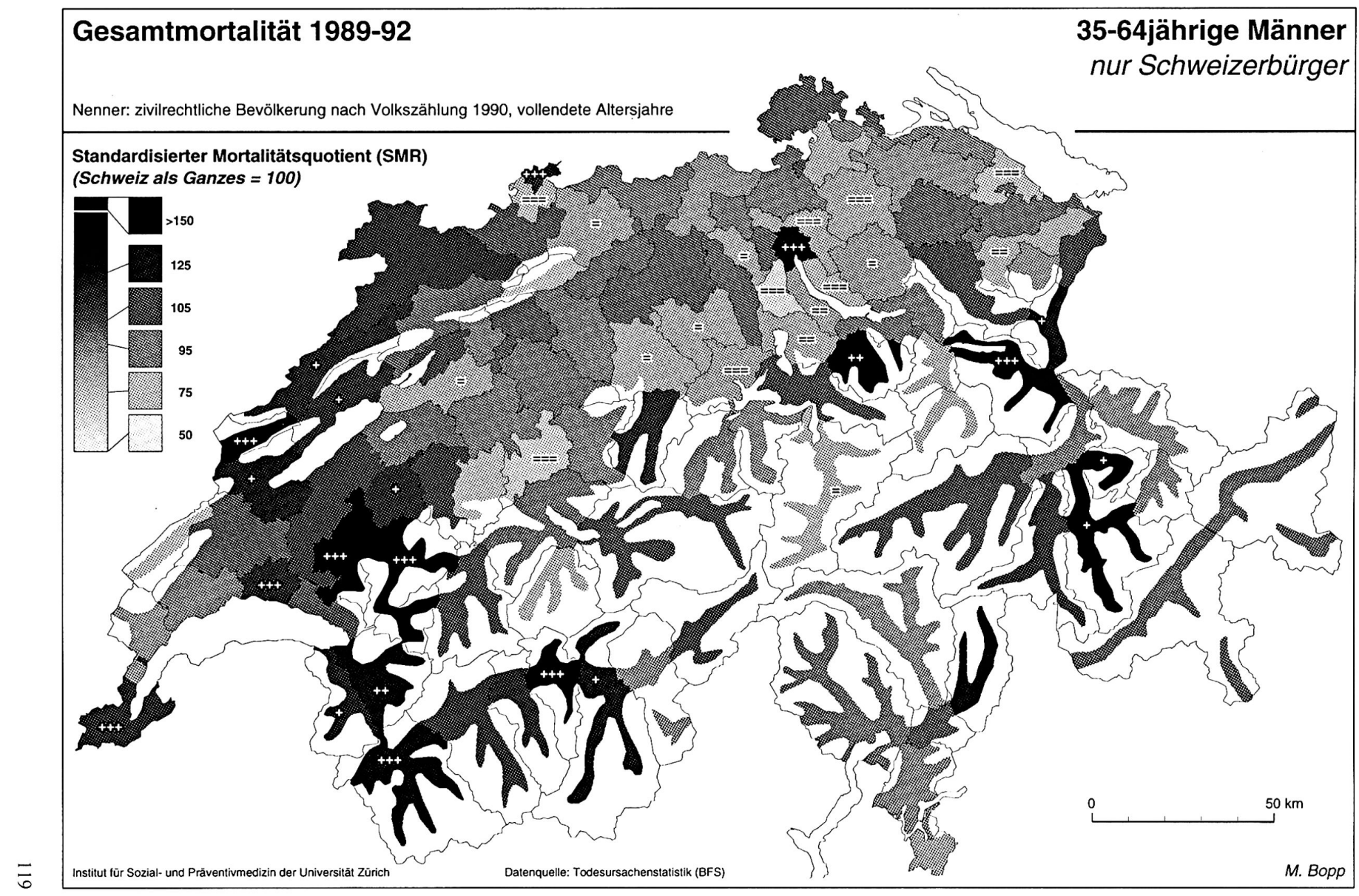


burgische und von dort über Aigle bis weit ins Wallis hinein

- in Basel-Stadt und Zürich. Auf Gemeindeebene war das relative Sterberisiko um 1990 in allen fünf Großstädten um 24-36\% höher als im Landesmittel, in auffälligem Kontrast zu Winterthur, St. Gallen und Luzern

- in einzelnen voralpinen Regionen der Deutschschweiz: v. a. Sarganserland/St. Galler Rheintal und Einsiedeln/Innerschwyz; langfristig zeigen auch die Nachbar- und Zwischenregionen March, Glarner Mittel- und Unterland sowie Innerrhoden Risikoerhöhungen

- in Teilen Graubündens, vorab Schanfigg und Misox (Mittelbünden ragt langfristig weniger deutlich hervor als um 1990).

Niedrige Mortalität - langfristig 15-25\% unterm Landesmittel - kennzeichnet das Umland der Großstädte, in scharfem Gegensatz zu den Zentren selbst: v. a. im Großraum Zürich und im Baselbiet. In Kontrast zur Karte für alle Alter und unter Einbezug der Ausländer zeigen sich für die Kantone Waadt und Genf Risikoerhöhungen von 8 bzw. 14\% gegenüber der Gesamtschweiz (einzig die Regionen Nyon, Morges/Rolle und La Vallée bleiben unterm Landesmittel). Eher unerwartet, aber langfristig bestätigt ist die günstige Position von Uri, während Oberthurgau und Außerrhoden um 1990 besonders gut abschneiden.

\section{Frauen (Abb. 3)}

Schon auf den ersten Blick erkennt man, daß die Kontraste flauer ausfallen als bei den Männern. Erhöhte Mortalität ist charakteristisch:

- entlang dem Jurabogen von Balsthal bis ins Val-deTravers, inkl. Biel/Seeland und längerfristig auch den Nordjura einbeziehend. Im Gegensatz zu den Männern ist die Risikoerhöhung in der Suisse romande nur angedeutet, v. a. im unteren Rhonetal, in La Sarine (Freiburg) und im Kanton Genf

- für die Großstädte Genf, Basel und Zürich, nicht aber für Bern

- im Voralpengebiet vom Kanton Schwyz bis zum Sarganserland, mit Fortsetzung in den Kanton Graubünden und einem Ausläufer ins Appenzellische; längerfristig rundet das St. Galler Rheintal diese Zone erhöhten Sterberisikos ab.
Niedrige Mortalität kann um 1990 im Umland der Großstädte nur noch angedeutet festgestellt werden (v. a. südlich der Stadt Zürich). Viel häufiger als bei den Männern zeigen hochalpine Regionen (Deutschwallis, Zentralalpen, Oberengadin) ein günstiges Bild, mit einem relativen Sterberisiko rund $20-25 \%$ unterm Landesmittel.

Im Gegensatz zur Karte für alle Alter und unter Einbezug der Ausländer zeigen sich für den Kanton Genf und die meisten Waadtländer Regionen Risikoerhöhungen; auch der Kanton Tessin schneidet nicht mehr so günstig ab.

\section{Diskussion}

Die Beurteilung der regionalen Unterschiede bei der Gesamtsterblichkeit bzw. der mittleren Lebenserwartung wird durch Zähler-Nenner-Probleme massiv erschwert. Maße, die alle Altersklassen und beide Geschlechter umfassen, sind als Indikator für die regionalen Lebensbedingungen kaum brauchbar.

Vor dem Hintergrund eines hohen Ausländeranteils, der langen Tradition der Schweiz als Immigrationsland sowie der überproportionalen Vertretung der Ausländer in Berufen mit bekannt hohem Sterberisiko (v. a. im Baugewerbe, vgl. GASS \& BOPP 1997) ist es kein Ruhmesblatt für unser Land, daß im Moment niemand in der Lage ist, seriöse Angaben über das Sterberisiko bzw. den Gesundheitszustand der ausländischen Wohnbevölkerung zu machen. Wohl resultiert über das ganze Land summiert der erwartete «healthy worker effect». Doch in etlichen Regionen wird für die ausländische Bevölkerung eine Mortalität im oder sogar über dem Landesdurchschnitt von Schweizern und Ausländern zusammen vermerkt; es handelt sich dabei vorwiegend um Berggebietsregionen, aber auch um die Großstädte Zürich, Basel und Bern. Aus den vorhandenen Daten kann nicht abgelesen werden, ob es sich hier um ein Zähler-Nenner-Problem, um selektive Wanderungseffekte oder etwa doch regional ungünstige Lebensumstände handelt.

Für die Schweizer Bürgerinnen und Bürger kann man für die Periode um 1990 die wichtigsten Verzerrfaktoren in den Griff bekommen, indem man sich für die

Zu Abb. 3 Relatives Sterberisiko der 35- bis 64jährigen Schweizer Frauen 1989-92 (siehe rechts).

Statistisch signifikante Abweichungen vom Landesmittel sind wie folgt gekennzeichnet:

$\begin{array}{ll}+ & \text { Risikoerhöhung signifikant auf dem 5\%-Niveau } \\ ++ & \text { Risikoerhöhung signifikant auf dem 1\%-Niveau } \\ +++ & \text { Risikoerhöhung signifikant auf dem 0,5\%-Niveau } \\ = & \text { niedrigere Mortalität signifikant auf dem 5\%-Niveau } \\ == & \text { niedrigere Mortalität signifikant auf dem 1\%-Niveau } \\ === & \text { niedrigere Mortalität signifikant auf dem 0,5\%-Niveau }\end{array}$




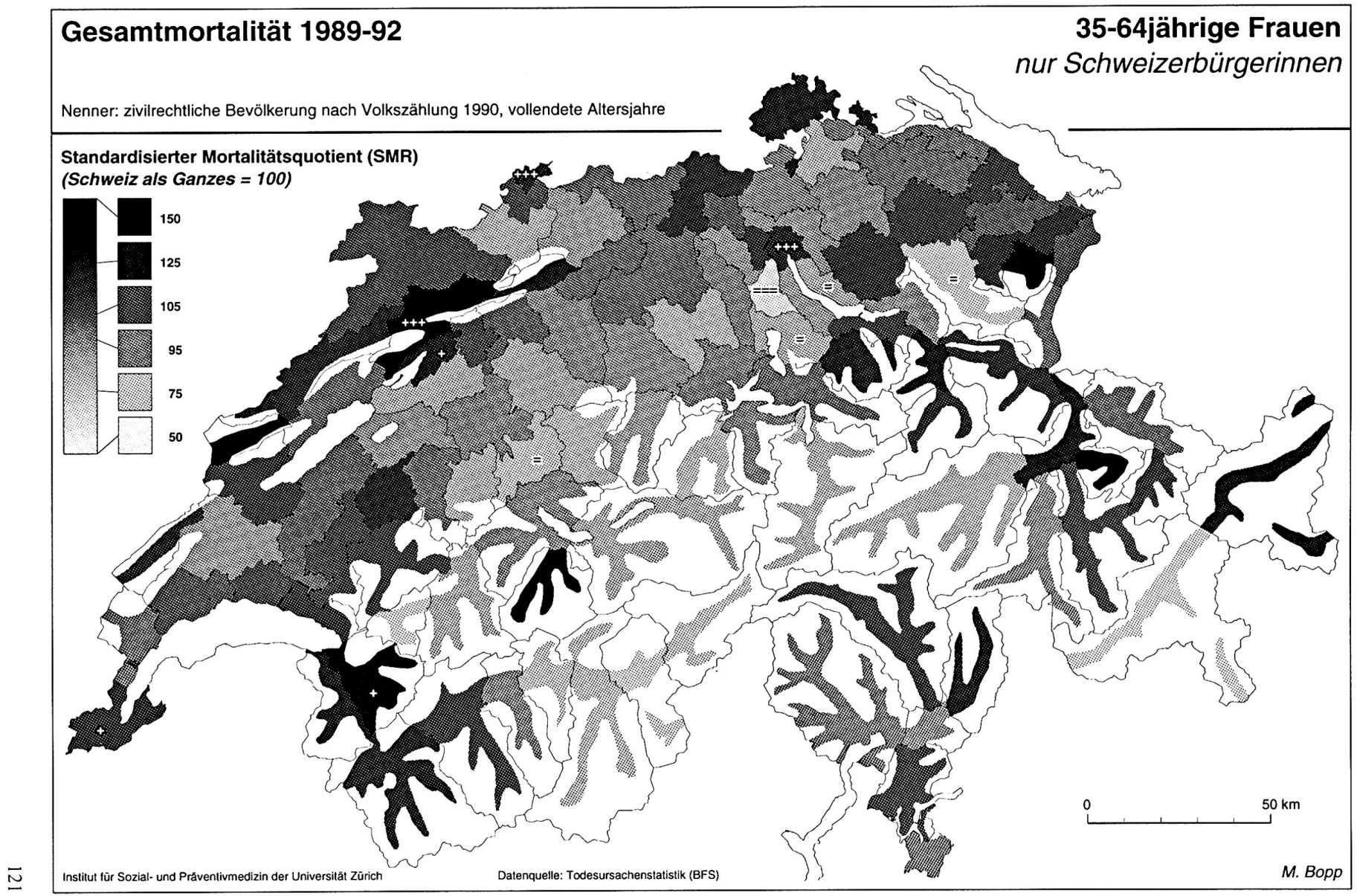


Wohnbevölkerung nicht auf die wirtschaftliche, sondern auf die zivilrechtliche Wohnsitzdefinition abstützt und alle Analysen für Männer und Frauen getrennt durchführt. Noch besser vergleichbar werden die Resultate, wenn man sich auf die mittleren Altersklassen beschränkt, denn hier werden die späteren regionalen Unterschiede in der Lebenserwartung vorgespurt. Zudem stellt der Gesundheitszustand von Personen im arbeitsfähigen Alter eine wichtige regionale Ressource dar. Das geographische Muster der Gesamtmortalität hat sich seit 1970 nur punktuell verändert. Generell hat die Peripherie aufgeholt und steht nun bei den 35- bis 64jährigen Frauen meist besser da als die Großstadtzentren und sogar als die wohlhabenden Umlandregionen.

Die im internationalen Vergleich hohe Lebenserwartung der Schweiz scheint ein günstiges Bild von den Lebensverhältnissen in unserem Land zu zeichnen. Bei genauerer Betrachtung der Altersgruppe 35-64 schälen sich jedoch ernstzunehmende Gesundheitsprobleme heraus:

- Für die unter 50jährigen Männer konnte seit 1970 kaum mehr eine Abnahme der Sterblichkeit verzeichnet werden, bei den 20- bis 39jährigen nimmt das Sterberisiko seit 1985 sogar zu.

- Die regionalen Unterschiede beim Sterberisiko und damit der Lebenschancen erscheinen in Anbetracht der heute nur noch geringen Unterschiede in der medizinischen Versorgung recht groß. Um 1990 war das alterskorrigierte Sterberisiko bei den 35- bis 64jährigen Schweizer Männern im Kanton Freiburg 50\% höher als in Baselland oder Uri. Dies entspricht einem Unterschied bei der mittleren Lebenserwartung von gut 2 Jahren (WANNER 1996). Auf regionaler Ebene wachsen die relativen Risikounterschiede auch unter Ausschluß der meist nur kurzlebigen Extremwerte auf 75 bis $100 \%$ bzw. zu einem Unterschied bei der mittleren Lebenserwartung von rund 4 Jahren an.

- Die geographischen Unterschiede sind bei den Männern deutlicher und persistenter. Sie können auch häufiger mit gesundheitsgefährdendem Verhalten in Einklang gebracht werden, v.a. mit Alkoholmißbrauch und hohem Unfallrisiko. Alkoholismus ist bei Männern im mittleren Alter die häufigste Diagnose beim Eintritt in die medizinische Abteilung von Allgemeinspitälern (SCHWEIZERISCHE FACHSTELLE FÜR ALKOHOL- UND ANDERE DROGENPROBLEME 1997). Hoher Alkoholkonsum gehört zu den wichtigsten Ursachen für vorzeitigen Tod (PETERSSON et al. 1984, ANDREASSON et al. 1988). Regressionsanalytisch kann die regionale Variation der Gesamtsterblichkeit der Männer in der Altersspanne 35-64 zu rund zwei Dritteln durch die Variation bei den alkoholassoziierten Diagnosen «erklärt» werden, obwohl diese nur einen Sechstel aller Todesfälle stellen (SCHÜLER \& BOPP 1997). Daneben spielt der durchschnittliche Reichtum einer Region (z. B. gemessen am Bundessteueraufkommen) und wohl auch das Ausmaß der Ungleichverteilung eine wichtige Rolle (WILKINSON 1986, SMITH 1996).
- Ein ungünstiges Bild präsentiert die Suisse romande - bei den Schweizer Männern mit einer durchschnittlichen Übersterblichkeit gegenüber dem Landesmittel von rund $14 \%$, bei den Frauen von durchschnittlich rund $8 \%$. Wegen des günstigeren Bildes bei den über 65jährigen und bei den Ausländern wird dieses Warnzeichen bei der Betrachtung von Gesamtsterbeziffern übersehen (SCHULER et al. 1997). Auch für die italienische Schweiz fällt das Bild für die 35- bis 64jährigen Schweizer bei weitem nicht so positiv aus wie für alle Einwohner zusammen.

- Die großen Agglomerationen entwickeln sich zunehmend zu den Gebieten mit den schärfsten Disparitäten: Während die Großstädte selbst zunehmend ungünstig dastehen, gehören die Sterberaten in ihrem Umland vor allem bei den Männern zu den niedrigsten im ganzen Land. Dies paßt zur selektiven Abwanderung von sozial höher gestellten Personen, die eine überdurchschnittliche Lebenserwartung besitzen. Aufgrund soziodemographischer Variablen wurde für die Schweiz eine mit der Agglomerationsgröße wachsende soziale Entmischung postuliert (DESSEMONTET \& RACINE 1996), die offenbar auch im Sterberisiko ihren Niederschlag findet. Daneben spielen vermutlich auch die Migrationsgewinne an sich eine Rolle, da gesunde Personen sich wohl eher einen Wohnsitzwechsel aufbürden (analog zum «healthy worker effect» könnte man von einem «healthy migrant effect» sprechen).

- Im europäischen Vergleich scheint die Lebenserwartung der Frauen stärker vom Reichtum eines Landes begünstigt zu werden als diejenige der Männer - mit der Schweiz weit vorne in der Rangliste. Innerhalb der Schweiz ist die günstige Position der wohlhabenden Regionen bei den Frauen relativ viel weniger ausgeprägt als bei den Männern. Ist dies Anzeichen für ein gesundheitsgefährdenderes Verhalten der Frauen in den «moderneren» Teilen des Landes, wie es in der Entwicklung der Lungenkrebsmortalität unübersehbar ist?

Eine sorgfältige Analyse regionaler Sterblichkeitsunterschiede ist nicht nur deshalb wichtig, weil es buchstäblich «ans Lebendige» geht, sondern auch deshalb, weil sie die kulturellen und sozialen Lebensbedingungen widerspiegeln. Da Mortalitätsraten sensitiv auf Änderungen solcher Rahmenbedingungen reagieren (CHENET et al. 1996), ist ihre ständige Beobachtung nicht nur für ein allgemeines Gesundheitsmonitoring, sondern für jedes regional ausgerichtete Interesse und Engagement unerläßlich.

\section{Anmerkung}

1 vgl. STATISTISCHES BUREAU DES EIDGENÖSSISCHEN DEPARTEMENTS DES INNERN (1914), STATISTISCHES BUREAU DES SCHWEIZERISCHEN FINANZDEPARTEMENTS (1916) und EIDGENÖSSISCHES STATISTISCHES BUREAU (1928). 


\section{Literatur}

ANDREASSON, S., ALLEBECK, P., ROMELSJÖ, A. (1988): Alcohol and mortality among young men: longitudinal study of Swedish conscripts. British Medical Journal 296, 1021-1025.

BISIG, B. \& PACCAUD, F. (1987): Geographische Verteilung wichtiger Todesursachen in der Schweiz 1969-72 und 1979-82. Amtliche Statistik der Schweiz, Nr. 151. Bern, Bundesamt für Statistik.

BOPP, M. (1989): Kartographie als Instrument in der Epidemiologie: Methodologische Überlegungen für den neuen Schweizer Krebsatlas. Sozial- und Präventivmedizin 34, 108-114.

BOPP, M. \& SCHÜLER, G. (1997): Neue Ansätze zur geographischen Mortalitätsanalyse. Nachweis langfristiger Krankheitshäufungen auch bei kleinen Fallzahlen. Forschung und Dokumentation Nr. 18. Zürich, Institut für Sozial- und Präventivmedizin der Universität Zürich.

BOUYER, J., et al. (1993): Epidémiologie - principes et méthodes quantitatives. Paris, Les Editions INSERM.

BOYLE, P., MUIR, C.S. \& GRUNDMANN, E. (1989): Cancer mapping. Recent Results in Cancer Research, No. 114.

BRESLOW, N.E. \& DAY, N.E. (1987): Statistical methods in cancer research. Volume II - The design and analysis of cohort studies. IARC Scientific Publications No. 82.

BROOKE, E.M. (1975): Géographie de la mortalité due au cancer en Suisse 1969-71. Lausanne: Institut universitaire de médecine sociale et préventive.

BUNDESAMT FÜR STATISTIK (1981): Sozialindikatoren für die Schweiz. Band 1: Gesundheit. Beiträge zur schweizerischen Statistik, Heft 81. Bern, BFS.

BUNDESAMT FÜR STATISTIK (1984): Geographische Verteilung der Krebssterblichkeit in der Schweiz 1979/81. Beiträge zur schweizerischen Statistik, Heft 119. Bern, BFS.

CHENET, L., OSLER, M., McKEE, M., KRASNIK, A. (1996): Changing life expectancy in the 1980s: why was Denmark different from Sweden? Journal of Epidemiology and Community Health 50, 404-407.

CLIFF, A.D., HAGGEIT, P. (1988): Atlas of disease distributions: analytic approaches to epidemiological data. Oxford, Basil Blackwell.

DESSEMONTET, P. \& RACINE, J.-B. (1996): Villes et agglomérations suisses. Geographica Helvetica 51, 143-168. EIDGENÖSSISCHES STATISTISCHES BUREAU (1928): Ehe, Geburt und Tod in der schweizerischen Bevölkerung während der Jahre 1901-1920. Schweizerische Statistische Mit- teilungen, X. Jahrgang, 4. Heft. Bern, Kommissionsverlag A. Francke.

GASS, R. \& BOPP, M. (1997): Berufsspezifische Mortalitätsrisiken der Männer in der Schweiz 1979-83. Statistik der Schweiz, Reihe 14: Gesundheit. Bern, BFS.

GUTZWILLER, F. \& JEANNERET, O. (Eds.) (1996): Sozial- und Präventivmedizin Public Health. Bern, Hans Huber.

HERZOG, A. (1988): Desktop Mapping - Desktop Publishing in der Kartographie, ein Anwendungsbeispiel. Geographica Helvetica 43, 21-26.

HOWE, G.M. (Ed.) (1986): Global geocancerology. A world geography of human cancers. Edinburgh, Churchill Livingstone.

LÜTZELER, R. (1994): Räumliche Unterschiede der Sterblichkeit in Japan. Sterblichkeit als Indikator regionaler Lebensbedingungen. Bonner Geographische Abhandlungen Heft 89. Bonn, Dümmler.

PETERSSON, B., TRELL, E., HENNINGSEN, N. C., HOOD, B. (1984): Risk factors for premature death in middle-aged men. British Medical Journal 288, 1264-1268.

SANS, S., KESTELOOT, H., KROMHOUT, D. (1997). The burden of cardiovascular diseases mortality in Europe. European Heart Journal 18, 1231-1248.

SCHÜLER, G. \& BOPP, M. (1997): Atlas der Krebsmortalität in der Schweiz 1970-1990. Basel, Birkhäuser.

SCHULER, M., BOPP, M., BRASSEL, K. \& BRUGGER, E. (1984): Strukturatlas Schweiz. Zürich, Ex Libris.

SCHULER, M., HUISSOUD, T., JEMELIN, CH., STOFER, S. (1997): Strukturatlas der Schweiz. Zürich, Verlag Neue Zürcher Zeitung.

SCHWEIZERISCHE FACHSTELLE FÜR ALKOHOL- UND ANDERE DROGENPROBLEME (1997): Zahlen und Fakten zu Alkohol und anderen Drogen. Lausanne, SFA.

SMITH, G. D. (1996): Income inequality and mortality: why are they related? British Medical Journal 312, 987-988.

STATISTISCHES BUREAU DES EIDGENÖSSISCHEN DEPARTEMENTS DES INNERN (1914): Graphisch-statistischer Atlas der Schweiz. Schweizerische Statistik, 191. Lieferung. Bern.

STATISTISCHES BUREAU DES SCHWEIZERISCHEN FINANZDEPARTEMENTS (1916): Ehe, Geburt und Tod in der schweizerischen Bevölkerung während der zehn Jahre 1891-1900. Fünfter Teil: Die Todesursachen. Schweizerische Statistik, 200. Lieferung. Bern, Kommissionsverlag A. Francke.

WANNER, P. (1996): Sterbetafeln für die Schweiz 1988-1993. Statistik der Schweiz, Reihe 1, Bevölkerung. Bern, Bundesamt für Statistik.

WILKINSON, R.G. (Ed.) (1986): Class and health - research and longitudinal data. London, Tavistock. 